Research Paper

\title{
Heyl promotes migration and invasion of melanoma cells via GRB2/PI3K/AKT signaling cascade
}

Yihuan $\mathrm{Pu}^{1}$, Mingxing Lei ${ }^{2,3}$, Yangmei Chen ${ }^{1}$, Yanran Huang4, Lingzhao Zhang1, Jiayi Chen ${ }^{1}$, Yujie Zhang', Xinyi Shao', Lin Liu ${ }^{1}$, Jin Chen ${ }^{1}$

1. Department of Dermatology, The First Affiliated Hospital of Chongqing Medical University, Chongqing 400016, China.

2. 111 Project Laboratory of Biomechanics and Tissue Repair, College of Bioengineering, Chongqing University, Chongqing 400044, China.

3. Key Laboratory of Biorheological Science and Technology of the Ministry of Education, College of Bioengineering, Chongqing University, Chongqing 400044, China.

4. Department of Orthopaedics, The First Affiliated Hospital of Chongqing Medical University, Chongqing 400016, China.

$\triangle$ Corresponding author: Jin Chen; E-mail: chenjin771010@163.com.

(C) The author(s). This is an open access article distributed under the terms of the Creative Commons Attribution License (https://creativecommons.org/licenses/by/4.0/). See http://ivyspring.com/terms for full terms and conditions.

Received: 2021.03.28; Accepted: 2021.09.18; Published: 2021.10.11

\begin{abstract}
Increasing evidence indicates that Notch signaling regulates multiple intracellular biological processes in malignant melanoma. Whereas how Notch signaling is transduced to influence melanoma cell behaviors remains largely elusive. Here we show that the Notch signaling downstream target Heyl promotes migration and invasion of melanoma cells via the GRB2/PI3K/AKT pathway. First, bioinformatics tools, immunohistochemistry, and Western blotting analysis showed that the expression of Heyl is increased in melanoma. Then, both in vivo and in vitro experiments showed that Heyl promotes the malignant behaviour of the melanoma cells. High-throughput RNA-sequencing analysis revealed that inhibition of Heyl results in decreased GRB2 expression in melanoma cells. Last, functional experiments confirmed that Heyl positively regulates GRB2/PI3K/AKT pathway to influence migration and invasion of melanoma cells. In summary, our results suggest that Heyl promotes the invasion and metastasis of melanoma cells by regulating GRB2/PI3K/AKT pathway. Our study provides potential therapeutics in tumor biology.
\end{abstract}

Key words: Melanoma, Hey1, GRB2/PI3K/AKT signaling, EMT, Invasion

\section{Introduction}

Melanoma is a highly malignant and aggressive grade of tumor with a high incidence rate, rapid growth, rapid metastasis, and high degree of resistance to radiotherapy and chemotherapy, leading to poor prognosis and high mortality. The primary treatment methods for melanoma include surgical resection, chemoradiotherapy, and immunotherapy [1].

The main signaling pathways involved in the occurrence and development of melanoma include MAPK, p13k-AKT, TGF- $\beta$, Wnt, and Notch signaling [2]. Previous studies have delineated a multifunctional role of Notch signaling, capable of controlling several aspects of melanoma pathogenesis. Notch signaling can synergistically interact with other signaling cascades such as Wnt, TGF- $\beta$, and ErbB signaling to influence melanoma cell behaviors [3].
Hey1, one of the target genes of the Notch signaling pathway, encodes a transcription factor with a wide range of functions [4]. Heisig et al. found that Hey1 modulates 507 genes involved in positive and negative transcription regulation, RNA splicing, chromatin assembly, protein transport, and organ development [5].

Hey1 plays important roles in regulating behaviors of various tumors. Tsuru et al. found that Hey1 expression was augmented in osteosarcoma, and Hey1 silencing inhibited the invasion and lung metastasis of this cancer [6]. In colon cancer, enhanced Hey1 expression is linked to the invasion of peripheral nerves and blood vessels and lymph node metastasis [7]. Park et al. found that Hey1 influenced the distant metastasis of breast cancer [8]. In addition, Hey1 was reported to promote the metastasis of lung 
cancer [9] and malignant progression of bladder cancer [10]. However, the molecular mechanism by which Hey1 regulates melanoma cell behaviors remains largely unknown. In the present study, we investigated the role of Hey1 in influencing human melanoma cells and elucidated that Hey1 promotes migration and invasion of melanoma cells via the GRB2/PI3K/AKT signaling pathway.

\section{Methods and materials}

\section{Tissue samples and cell culture}

Twenty-two normal skin tissues and twenty-seven melanoma tissues were collected from the Department of Dermatology, the First Affiliated Hospital of Chongqing Medical University. No patients had undergone radiotherapy or chemotherapy before the operation and all patients provided written informed consent before surgery. Utmost care was taken to ensure that the privacy rights of human participants were safeguarded. Specimen collection was approved by the clinical ethics committee of the First Affiliated Hospital of Chongqing Medical University and all procedures complied with the Declaration of Helsinki. The melanoma cell lines mel-888, mel-624, A375, and B16 were gifted by Dr. Tongchuan He (the University of Chicago Medical Center, Chicago, USA). The gll-19 cell line was donated by Dr. Qu Le (China Medical University, Shenyang, China). The human normal melanocyte cell line PIG1 was gifted by Dr. Chunying Li (Fourth Military Medical University, Shanxi, China). Melanoma cell lines were cultured in Dulbecco's Modified Eagle's Medium (HyClone, Logan, UT, USA) supplemented with $10 \%$ fetal bovine serum (Thermo Fisher Scientific, Waltham, MA, USA), 1\% penicillin, and $1 \%$ streptomycin. PIG1 cells were cultured in 254 medium supplemented with human melanocyte growth supplement (Thermo Fisher Scientific), 5\% fetal bovine serum (Thermo Fisher Scientific), $1 \%$ penicillin, and $1 \%$ streptomycin. All cells were cultured in a $5 \% \mathrm{CO} 2$ incubator at $37^{\circ} \mathrm{C}$.

\section{Immunohistochemistry}

Immunohistochemistry (IHC) analysis was performed using paraffin-embedded sections. An Immunohistochemistry sp-9000 Kit (Beijing Zhongshan Jinqiao Biotechnology Co., Ltd., Beijing, China) was used to stain and analyse all sections. The intensity of immunohistochemical staining and the average percentage of positive cells were evaluated.

\section{Gene Expression Profiling Interactive Analysis}

The GEPIA online database (http://GEPIA. cancer-pku.cn/) was used to analyse the mRNA expression of Hey1 in melanoma. GEPIA samples were obtained from the Cancer Genome Atlas and Genotype Tissue Expression projects.

\section{Small interfering RNA transfection and adenovirus infection}

The control small interfering RNA (siRNA) (s20c-0600) and Hey1 siRNA (sc-37913) were purchased from Biomics Biotech (Jiangsu, China). All siRNA transfection experiments were carried out according to the manufacturer's procedures using Lipofectamine RNAiMAX (Life Technologies, Carlsbad, CA, USA). Dr. Luo (The First Affiliated Hospital of Chongqing Medical University, Chongqing, China) gifted Hey1 overexpression (Ad-Hey1) and interference Hey1 recombinant adenovirus, as well as red and green fluorescent protein recombinant adenovirus as controls. Adenoviruses were transfected into A375 cells with polyethylene (Sigma Aldrich, St. Louis, MO, USA). Measurement of the fluorescence intensity $24 \mathrm{~h}$ later indicates that transfection was successful.

\section{Western blotting}

The protein extract was separated by $10 \%$ sodium dodecyl sulphate-polyacrylamide gel electrophoresis and then transferred to the polyvinylidene fluoride (PVDF) membranes. The membrane was placed in a blocking solution (Shanghai Biyun Tian Biotechnology Co., Ltd., Shanghai, China) for $2 \mathrm{~h}$ at 37 ${ }^{\circ} \mathrm{C}$. Next, the membrane was incubated overnight with the target primary antibody Hey1 $(1: 1,000$; Abcam, Cambridge, MA, USA), E-cadherin (1:5,000; Abcam), GRB2 (1:1,000; Abcam), N-cadherin (1:1,000; Abcam), matrix metalloproteinase-2 (MMP-2, 1:1,000; Abcam), MMP-9 (1:1,000; Cell signaling Technology, Danvers, MA, USA), MMP-7 (1:1,000; Cell signaling Technology), Snail (1:1,000; Cell signaling Technology) vimentin (1:1,000; Cell signaling Technology), PI3K (1:1000; Cell signaling Technology), Phosphorylated PI3K (1:1000; Affinity Biosciences, Jiangsu, China), AKT (1:1000; Cell signaling Technology), Phosphorylated AKT (ser308, 1:1000; Cell signaling Technology), and Phosphorylated P21 (1:1000; ImmunoWay Biotechnology, Plano, TX, USA), then washed with Tris-buffered saline containing Tween 20 three times, followed by incubation with an appropriate secondary antibody (1:5000; Beijing Zhongshan Jinqiao Biotechnology Co., Ltd., Beijing, China) at 37 ${ }^{\circ} \mathrm{C}$ for $1 \mathrm{~h}$. Protein bands were visualised using the SuperSignal West Pico chemiluminescence substrate Kit (EMD Millipore, Billerica, MA, USA). Each experiment was repeated thrice.

\section{Cell proliferation assay}

Cell viability was detected by CCK 8 assay. 3,000 
cells were seeded into each well of 96-well plates. After $0,24,48,72$, or $96 \mathrm{~h}$ of culture, CCK8 solution (Cell Counting kit-8, Dojindo, Kumamoto, Japan) was added to each well and the cells were further incubated at $37^{\circ} \mathrm{C}$ for $1 \mathrm{~h}$. The optical density (OD) was measured at $450 \mathrm{~nm}$ with a microplate reader.

\section{Colony-formation assay}

Log-phase cells were seeded into 6 well-plates at a density of 800 cells/well and incubated until clones were observed. The cells were then fixed with $4 \%$ paraformaldehyde and stained with $0.1 \%$ crystal violet. Visible colonies were counted.

\section{Migration, invasion, and wound-healing analysis}

A Transwell chamber (24-well Transwell chamber, $8 \mu \mathrm{m}$; Corning, Inc., Corning, New York, USA) was used for migration and invasion analysis. At $48 \mathrm{~h}$ after transfection, the cells were re-suspended in the serum-free medium, and then cells at a density of $50,000 / 200 \mu \mathrm{L}$ were inoculated into the upper chamber. High-glucose medium containing $10 \%$ fetal bovine serum was added to the lower chamber. The cells were cultured for $24 \mathrm{~h}$ and stained with $0.1 \%$ crystal violet. The Transwell invasion assay was performed similarly to the migration assay, but the bottom of the Transwell chamber was coated with Matrigel at a dilution of 1:10. In wound-healing analysis, the cells were seeded into a 6-well plate. When the transfected cells grew to $90 \%$, a $200 \mu \mathrm{L}$ pipette was used to scratch the wound, and the cells were incubated for $24 \mathrm{~h}$. Specific scratch areas were photographed at 0 and $24 \mathrm{~h}$. Cell migration was evaluated by mobility: (original scratch width - new scratch width)/original scratch width $\times 100 \%$. Each experiment was repeated thrice.

\section{High-throughput sequencing analysis}

Total RNA was isolated using the RNeasy mini kit (Qiagen, Germany). Paired-end libraries were synthesized by using the TruSeq ${ }^{\mathrm{TM}}$ RNA Sample Preparation Kit (Illumina, USA) following Sample Preparation Guide. The cleaved RNA fragments are copied into first-strand cDNA using reverse transcriptase and random primers. This is followed by second-strand cDNA synthesis using DNA Polymerase I and RNase $\mathrm{H}$. These cDNA fragments then go through an end repair process, the addition of a single ' $A$ ' base, followed by ligation of the adapters. The products are next purified and enriched with PCR to create the final cDNA library. Purified libraries were quantified by the Qubit ${ }^{\circledR} 2.0$ Fluorometer (Life Technologies, USA) and validated by the Agilent 2100 bioanalyzer (Agilent Technologies, USA) to confirm the insert size and calculate the mole concentration.
Cluster was generated by cBot with the library diluted to $10 \mathrm{pM}$ and then was sequenced on the Illumina NovaSeq 6000 (Illumina, USA).

\section{Immunofluorescence}

Immunofluorescence (IF) analysis was performed using paraffin-embedded sections. Paraffin sections were dehydrated in sequential order. Auto-fluorescence was quenched with BSA. The samples were incubated with primary antibodies and secondary antibodies (Alexa 488 and FITC, Servicebio, Wuhan, China) at a dilution of 1:5000, and then mounted by DAPI staining reagent (Servicebio). Samples were visualized under a Pannoramic Scanner system (Pannoramic DESK, P-MIDI, P250, 3D HISTECH, Hungary).

\section{Xenotransplantation in nude mice}

Approximately $5 \times 10^{6}$ adenovirus-transfected A375 cells were suspended in $100 \mu \mathrm{L}$ serum-free medium and then injected into nude mice. Female nude mice were employed in the study. The tumor size was recorded with a Vernier caliper every week, and the tumor volume was calculated using the following formula: length $\times$ width $2 \times 0.5$. Four weeks later, the mice were sacrificed by cervical disc dislocation. Tumour tissue and lung tissue were collected and embedded in paraffin for IHC and hematoxylin and eosin analysis. All procedures related to animal handling, care, and treatment were complied with the ARRIVE guidelines and were carried out in accordance with the National Institutes of Health guide for the care and use of laboratory animals. The scheme was also approved by the Animal Ethics Committee of the First Affiliated Hospital of Chongqing Medical University.

\section{Statistical analysis}

Data are expressed as the mean \pm standard deviation, and all values were analysed using GraphPad Prism (Prism 8.0.2; GraphPad Software, Inc., La Jolla, CA, USA). The student's t-test was used to evaluate the differences between the experimental group and control group. $\mathrm{P}<0.05$ was considered to indicate statistically significant results.

\section{Results}

\section{Heyl expression was increased in human melanoma tissues and melanoma cells}

Hey1 expression was first examined in normal human skin and melanoma tissues by IHC. The Hey1 expression level was higher in melanoma tissue than in normal skin tissue (Fig. 1A). Samples were scored on the basis of immunoreactivity scores, negative (14) and positive (5-12) [11]. The positivity rate of Hey1 
was $18 \%(4 / 22)$ in normal skin and $77 \%(21 / 27)$ in melanoma tissue (Fig. 1B). The expression of Hey1 protein was significantly higher in melanoma cells (gll-19, mel-888, mel-624, A375, and B16) than that in the PIG1 cells (Fig. 1C). The Hey1 mRNA expression level was significantly upregulated in melanoma tissues compared to the corresponding expression level in normal skin tissues (Fig. 1D). As the A375 cell line is widely used for melanoma investigations, it was also employed herein to examine the role of Hey1 in melanoma.

\section{Heyl promoted the invasion, migration, and EMT process of melanoma cells}

Hey1 protein expression was first examined in adenovirus- and siRNA-treated A375 cells by Western blotting. Its expression level was significantly elevated in the overexpression group and decreased in the interference group, compared to the expression level in the corresponding control group (Fig. 2A). Then, the CCK8 assay (Fig. 2B) and Colony-forming assay (Fig. 2C) were used to detect the impact of Hey1 on the proliferation of A375 cells. The results showed that Hey1 did not significantly affect the proliferation of A375 cells. However, wound-healing analysis (Fig. 2D) and Transwell invasion assay (Fig. 2E) results showed that the migration and invasion abilities were significantly enhanced in the Heyl overexpression group and inhibited in the Hey1 interference group, when compared to the corresponding control groups.
A
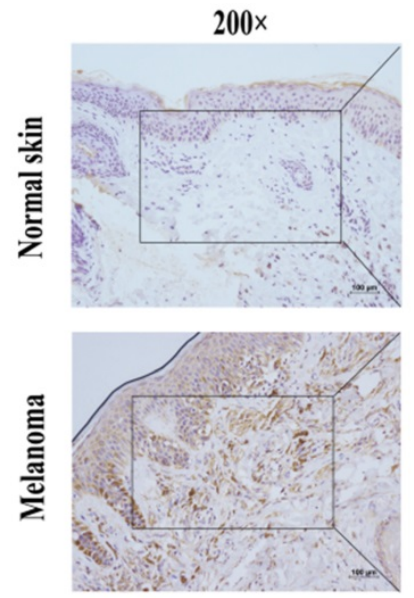

C

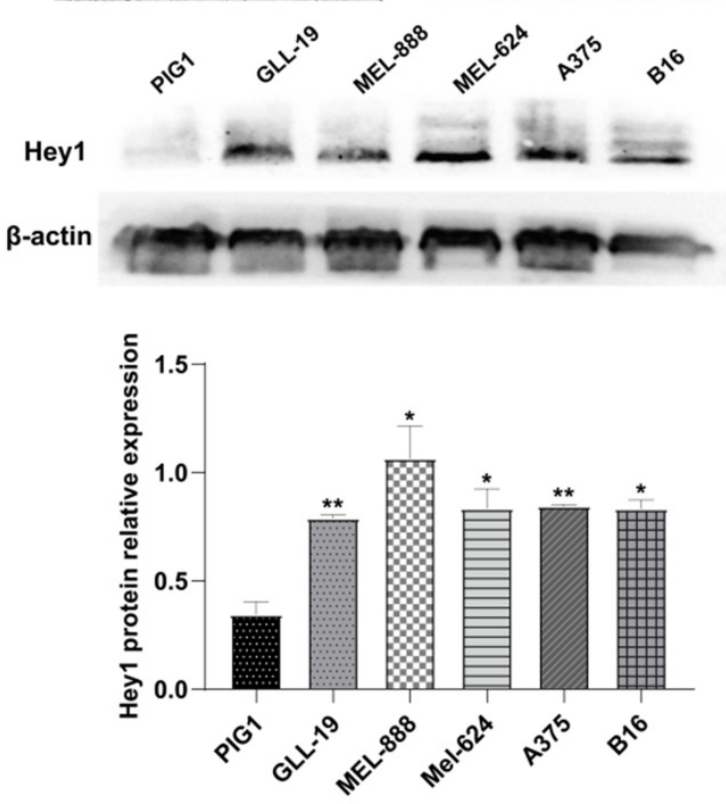

$400 x$
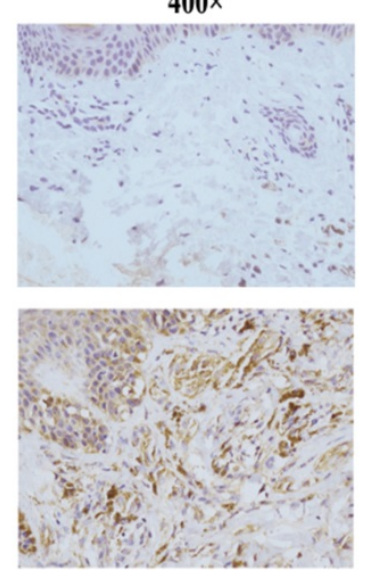

(1)

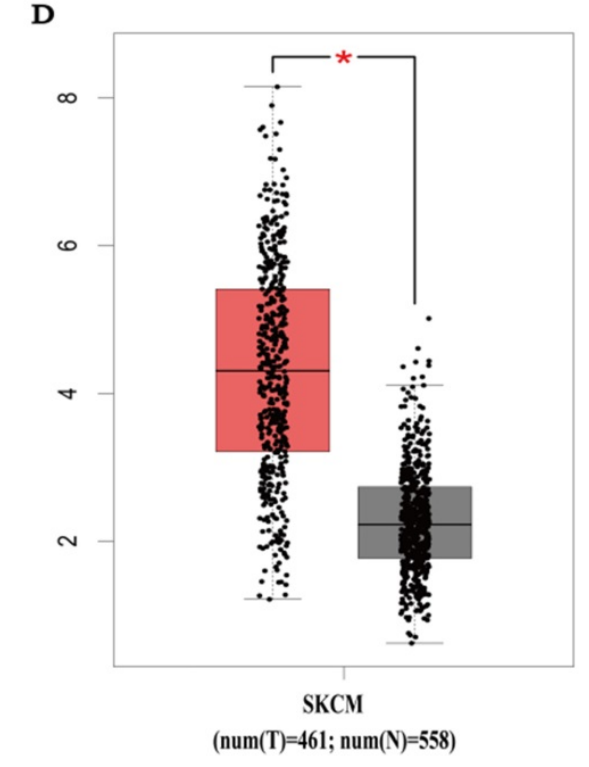

Figure 1. Heyl expression is upregulated in melanoma tissues and melanoma cell lines. (A) Representative immunohistochemical staining of Heyl in normal skin $(n=22)$ and melanoma tissue $(n=27)$ paraffin sections. (B) The percentage of Heyl-negative and -positive staining scores in normal skin and melanoma tissues. (C) Heyl expression was measured by Western blotting analysis in human epidermal melanocytes PIGl cell line and melanoma cell lines. Data are shown as mean $\pm \mathrm{SD}$. $* \mathrm{P}<0.05$, $* * \mathrm{P}<0.01$, $* * * P<0.001$. (D) Heyl expression in normal skin $(n=558)$ and skin cutaneous melanoma $(n=461)$ in the GEPIA database. Abbreviations: GEPIA, Gene Expression Profiling Interactive Analysis; Heyl, Hes related family bHLH transcription factor with YRPW motif 1. 
A

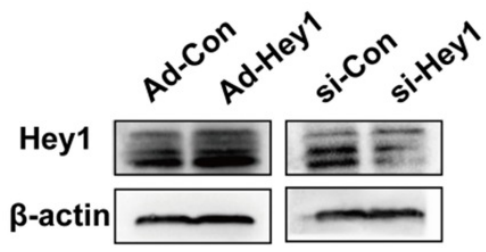

C
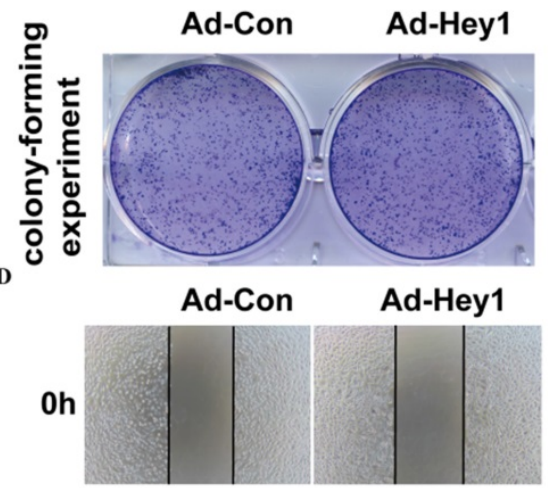

$24 \mathrm{~h}$

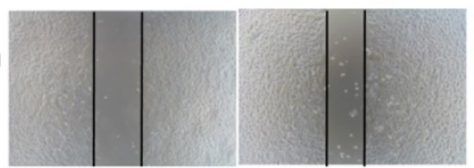

E

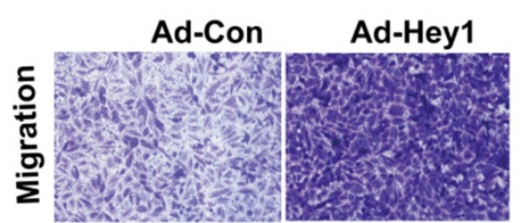

Ad-Con

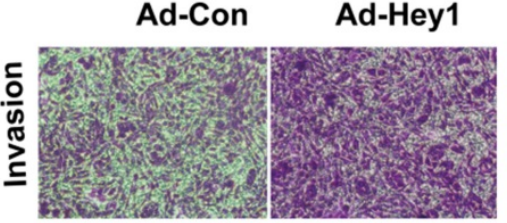

B

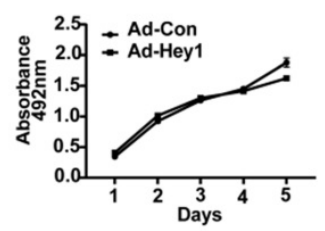

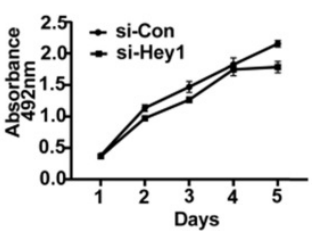

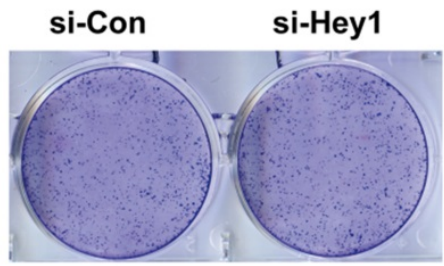

si-Hey1
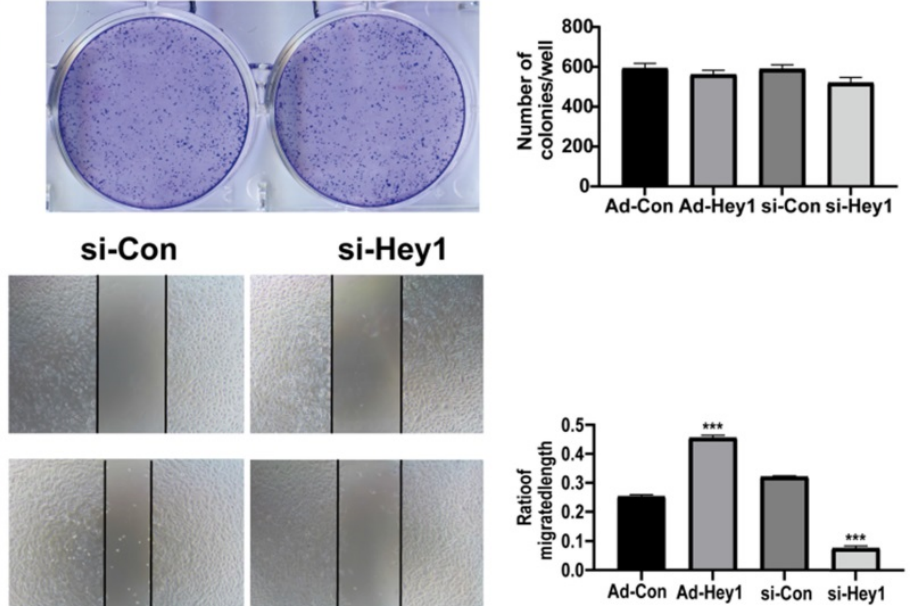

Figure 2. Heyl does not alter cell proliferation, but promotes the invasion and migration of melanoma cells. (A) Western blotting analysis of the Heyl expression levels in Heyl siRNA-treated A375 cells and in Ad-Heyl-infected A375 cells. (B) Cell viability was measured by the CCK8 assay in A375 cells. (C) Images of colony formation and depiction of the colony formation rates. (D) The representative images (magnification $\times 200$ ) and percentages of cell migration. (E) The representative images (magnification $\times 200$ ) and the number of migrated and invaded cells. Data are shown as mean $\pm \mathrm{SD}$. $* \mathrm{P}<0.05, * * \mathrm{P}<0.01, * * * \mathrm{P}<0$. 001 . Data represent three independent experiments. Abbreviations: Con: control; si: small interfering RNA; Ad: recombinant adenoviruses; CCK8, 2 - (2-methoxy-4-nitrophenyl) - 3 - (4-nitrophenyl) - 5 (2,4-disulfonic acid benzene) - $2 \mathrm{H}$ tetrazole monosodium salt; EMT, epithelial-mesenchymal transition.

Epithelial-mesenchymal transition is an important step in early tumor metastasis [12]. The protein matrix metalloproteinases (MMPs) can degrade the extracellular matrix to promote tumor invasion [13]. Previous studies have shown that Hey1 can promote the EMT process and the expression of matrix metalloproteinases expression profile of tumor cells [14-16]. Here, Western blotting was used to detect the impact of Hey1 on the expression of EMT-related proteins in A375 cells. The results showed that the expression of E-cadherin was significantly increased and the expression of N-cadherin, MMP-2, MMP7, MMP-9, Vimentin, and Snail was significantly decreased in the Hey1 interference group, compared to that of the controls.
On the contrary, the expression of E-cadherin was significantly decreased and the expression of N-cadherin, MMP-2, MMP7, MMP-9, Vimentin, and Snail was significantly increased in the Hey1 overexpression group, compared to that of the controls (Fig. 3A-B).

\section{Heyl promoted the growth and lung metastasis of melanoma cells in nude mice}

A xenograft animal model was used to study the effect of Hey1 on the growth and metastasis of melanoma cells in vivo. Tumour volume was measured once per week. Compared with the tumor volume in the control group, the tumor volume was significantly reduced in the Hey1 interference group 
but was significantly increased in the Hey1 overexpression group (Fig. 4A-C). Subsequently, we collected lung tissues from tumorigenic mice for hematoxylin and eosin staining. Compared with the control group, there were smaller and fewer metastatic tumor islands in the Hey1 interference group and larger and more metastatic tumor islands in the Hey1 overexpression group (Fig. 4D). These results demonstrated that Hey1 promotes the metastasis and invasion of melanoma cells in vivo.

\section{Hey1 interference negatively regulated GRB2 expression and inhibited the PI3K/AKT signaling pathway in $\mathbf{A} 375$ cells}

High-throughput sequencing was performed to characterize the effector molecules regulated by Hey1. A total of 232 coding genes were identified and visualised in a scatter plot (Fig. 5A). Kyoto Encyclopedia of Genes and Genomes (KEGG) analysis was performed on differential gene expression and the top30 were displayed (Fig. 5B). Since p53 and FoxO signaling pathways were largely studied in melanoma cells $[17,18]$, we selected the ErbB signaling pathway for further verification. Among ErbB signaling pathway genes (Supplementary 1), we observed that the mRNA level of the adaptor protein GRB2 was significantly decreased after inhibition of Hey1 in melanoma cells, compared to that of the control. Then, we performed double immunofluorescence labeling of them in melanoma tissues. Intriguingly, in addition to Hey1, we observed GRB2 expression was also significantly increased in the melanoma tissues compared to that of the normal skin tissues (Fig. 5C). These results indicate that Hey1 may positively regulate GRB2 expression in melanoma tissue.

Previous study showed that GRB2 can activate PI3K/AKT signaling in colorectal cancer cells [19] and the PI3K/AKT pathway regulates cell differentiation, migration, and invasion [20]. In the present study, KEGG analysis showed that among the PI3K/AKT signaling, and the phosphorylation level of the P21 target gene was significantly decreased in the Hey1 inhibition group, compared to that of the control group. Thus, Western blotting was used to detect the expression of key proteins in the GRB2/PI3K/AKT signaling cascade. The results showed that the total AKT and PI3K protein expression did not significantly change, However, GRB2, p-PI3K, p-AKT (ser308), and p-P21 protein expression was significantly decreased in the Hey1 inhibition group, compared to that of the controls (Fig. 6A-B). In contrast, the expression of these proteins was significantly increased in the Heyl overexpression group, compared to that of the control group. These results indicated that Hey1 regulates melanoma cell behavior through GRB2/PI3K/AKT signaling pathway (Fig. 7).
A

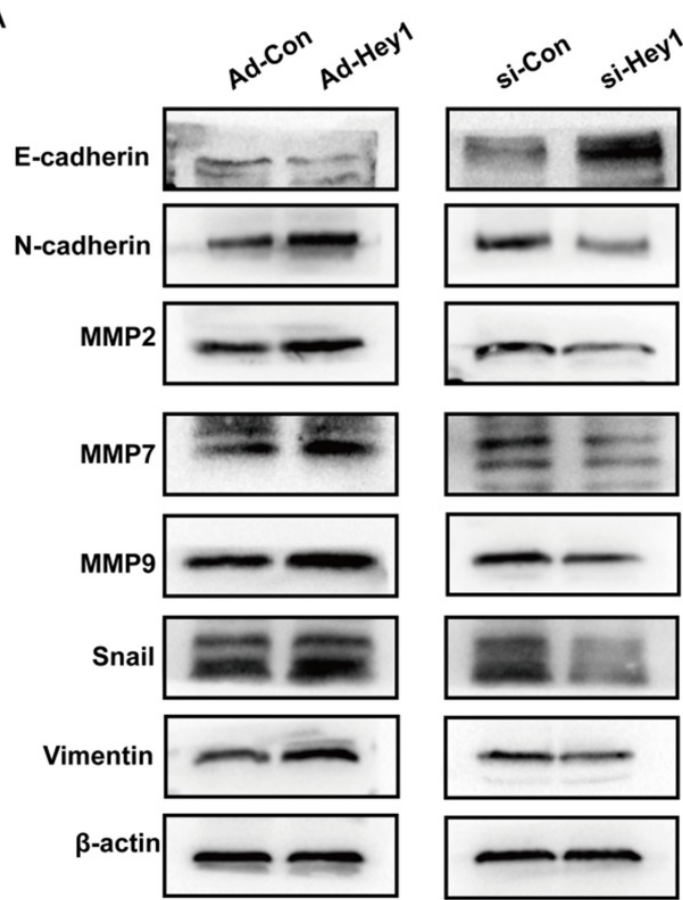

B
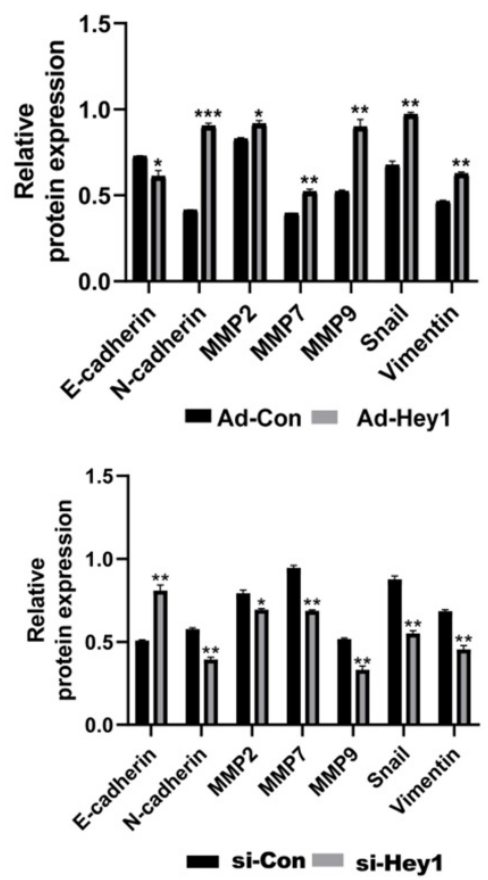

Figure 3. Hey1 promotes EMT and MMPs protein expression of melanoma cells. (A) Western blotting analysis of protein expression levels of E-cadherin, $\mathrm{N}$-cadherin, MMP-9, MMP-7, MMP-2, vimentin, and Snail. (B) Relative expression of each protein to $\beta$-actin in A375 cells. Data are shown as mean \pm SD. $* P<0.05$, $* * P<0.01$, $* * * P<0.001$. Data represent three independent experiments. Abbreviations: EMT, epithelial-mesenchymal transition; MMP: matrix metalloproteinase. 
A

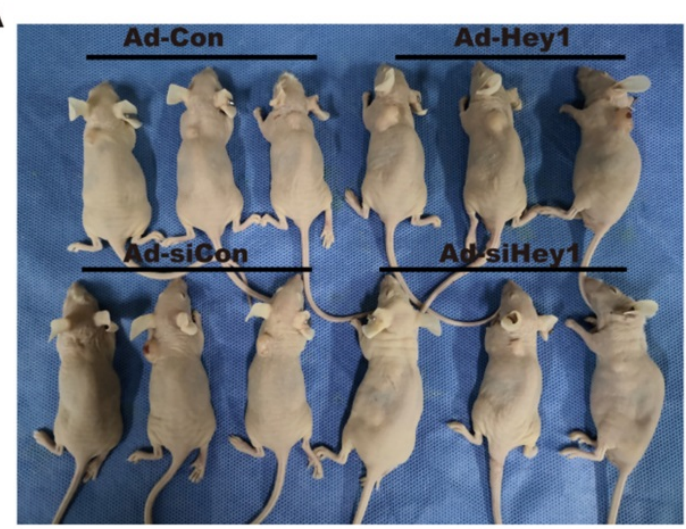

C

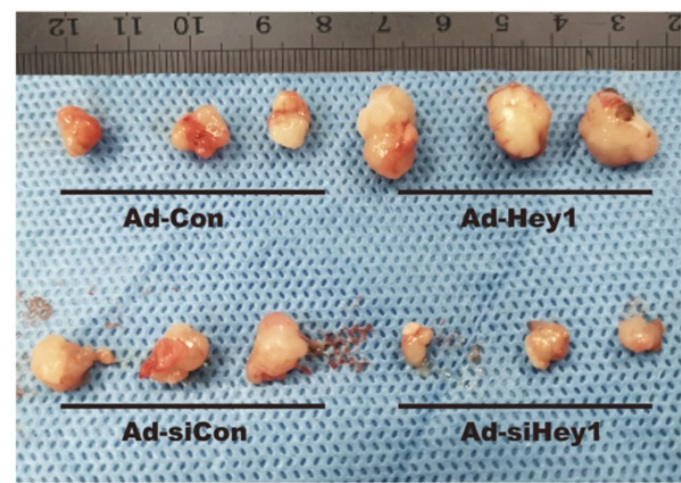

B
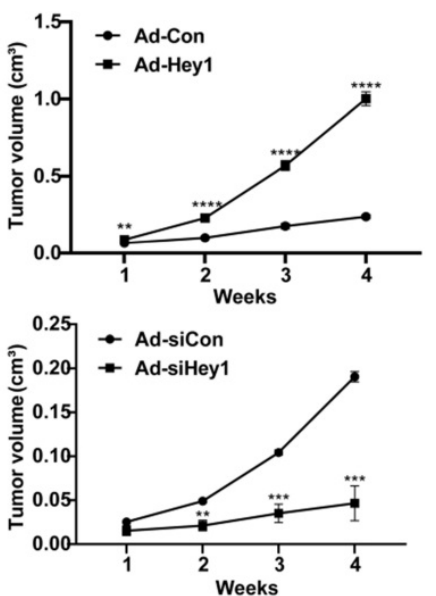

D
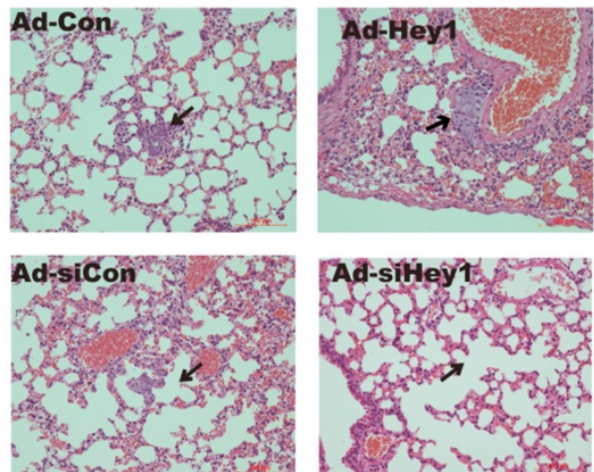

Figure 4. Heyl interference suppresses and Heyl overexpression promotes tumor growth and lung metastases of A375 cells in vivo. (A) These graphs show the tumor xenografts 4 weeks after ectopic-subcutaneous implantation in nude mice. (B) Tumour growth curve. (C) Representative images of primary implanted tumor mass of mice sacrificed at 4 weeks. (D) Representative hematoxylin and eosin images of lung specimens. Black arrow indicates metastatic lung nodule. Data are shown as mean \pm SD. $* \mathrm{P}<0.05, * * \mathrm{P}<0.01$.

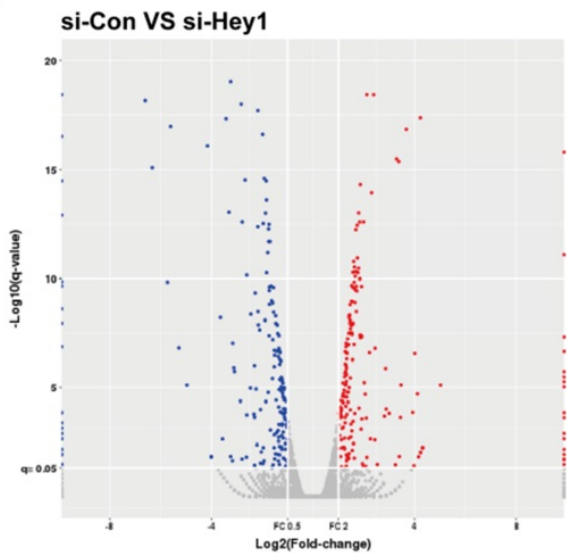

C

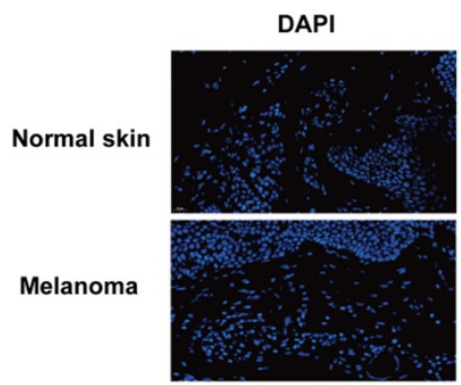

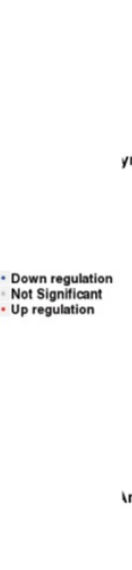

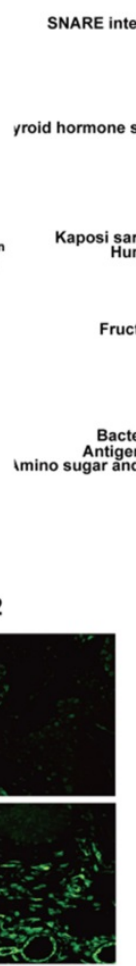

ons in vesicular transpos Restions in vesicular transport
RNA degradation
Renal cell carcioma
Relaxin signaling pathway
Pyrimidine metabolism Proteoglycans in cancer Prostate cancer
esis, secretion and action
Pancreatic cancer
p53 signaling pathway

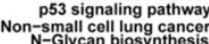
Morphine addiction Mitophagy - animal
ssociated herpesvirus

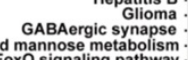
FoxO signaling pathway
ErbB signaling pathway Endometrial cance
Colorectal cancer Chronic myeloid leukemia
Cholinergic synapse Bacterial invasion of epithelial cells Top 30 of Pathway Enrichment

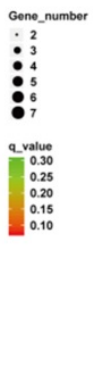

Figure 5. High-throughput sequencing results of A375 interfering with Heyl and immunofluorescence results of melanoma tissues. (A) Volcano plot shows the gene expression in si-Heyl A375 cells and control cells, and 232 differentially expressed genes (>2-fold change) were identified. (B) Top 30 genes identified in KEGG pathway enrichment. (C) The Heyl and GRB2 protein expression levels were detected in melanoma tissues by immunofluorescence. Abbreviations: si: small interfering RNA; KEGG: Kyoto Encyclopedia of Genes and Genomes. 


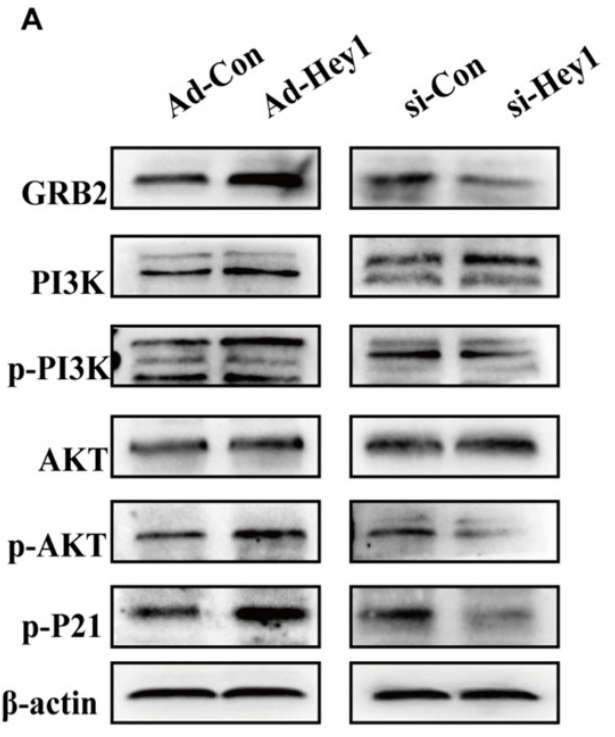

B
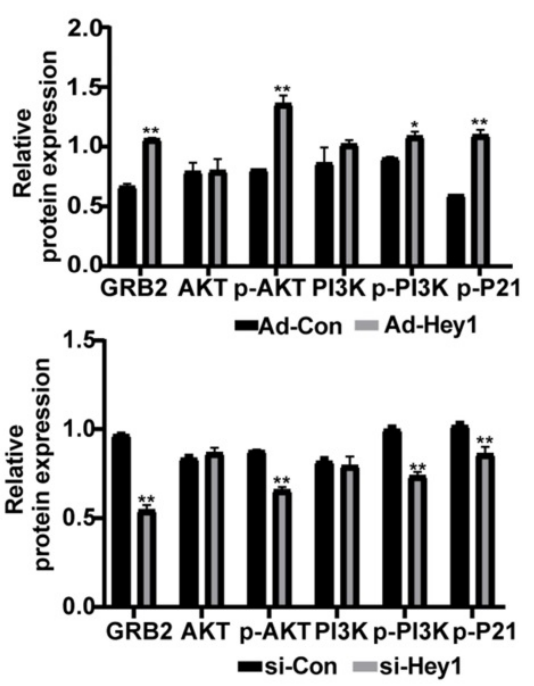

Figure 6. Hey1 interference negatively regulates GRB2 expression and attenuates the GRB2/PI3K/Akt signaling pathway in A375 cells. The protein expression levels of GRB2, PI3K, p-PI3K, AKT, p-AKT, and p-P21, as well as the relative expression of each protein to $\beta$-actin, compared with the corresponding control expression levels. (A) Akt and PI3K levels were not significantly changed. GRB2, p-PI3k, p-AKT (ser308), and p-P21 levels were significantly decreased in the A375-interference group. The corresponding expression levels noted in the overexpression group showed an opposite pattern. Data are shown as mean $\pm \mathrm{SD}$. $* \mathrm{P}<0.05$, $* * \mathrm{P}<0.01, * * * \mathrm{P}<0.001$. Data represent three independent experiments.

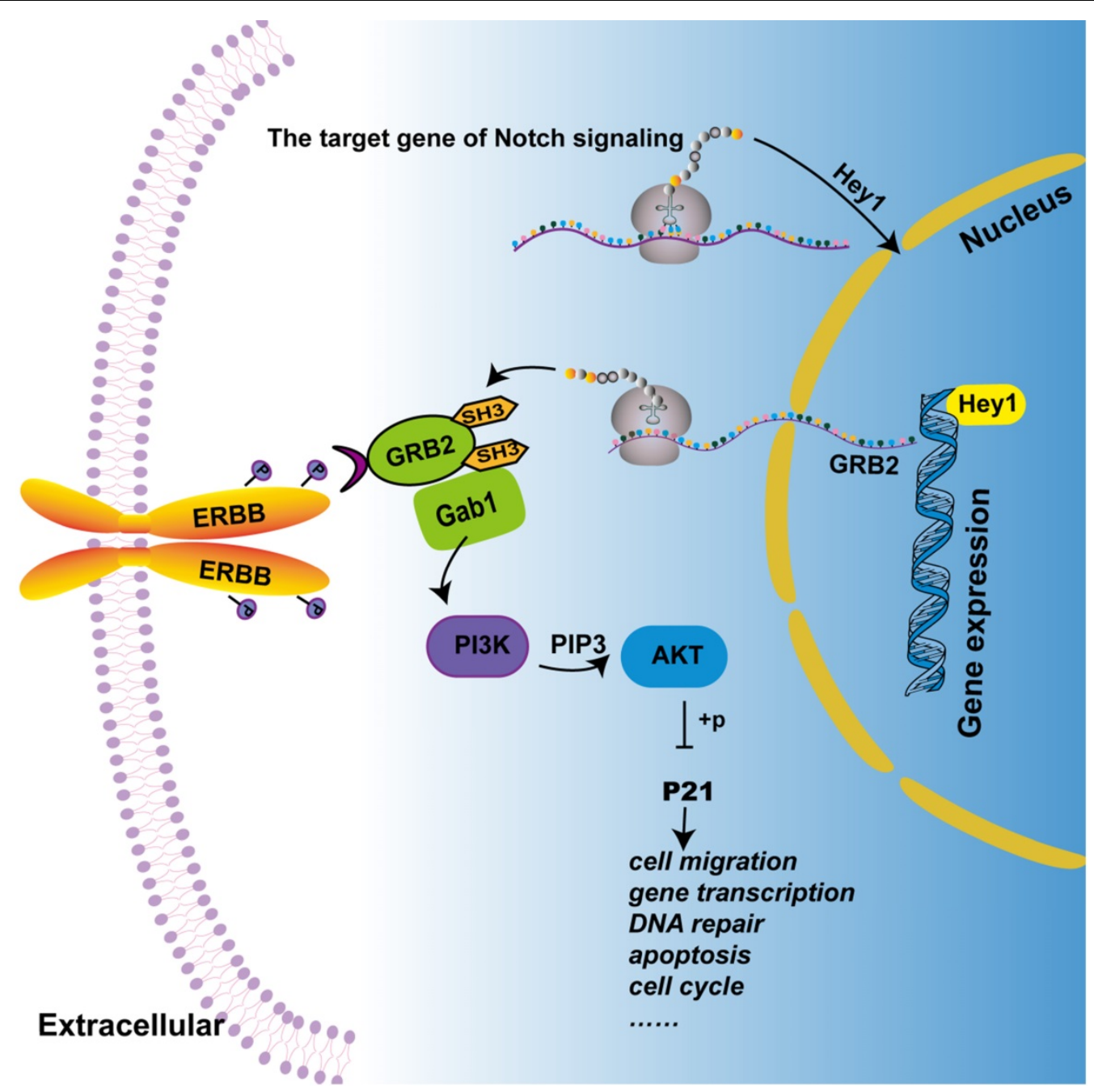

Figure 7. Hey1 regulates the PI3K/Akt signaling pathway by regulating GRB2 expression in melanoma cells. Combined with the previous and the present study, we depict that Heyl promotes the expression of GRB2. GRB2 recruits and activates phosphatidylinositol-3-kinase (PI3K) to produce second messenger PIP3 by binding with Gabl (GRB2 associated binding protein). PIP3 then phosphorylates and activates AKT, which acts on P21, thus affecting the biological process of the tumor. 


\section{Discussion}

Significant progress has been made in treating melanoma in the past decades. However, melanoma remains a lethal cancer type, especially in the late stages of diagnosis. Thus, investigation of the progression of melanoma is positioned as a very important issue in tumor biology. In the present study, we investigated the crucial role of Hey1, a target gene of Notch signaling, on the biological function of melanoma and its molecular mechanisms. We found that Hey1 mediates GRB2 to regulate PI3K/ AKT signaling in melanoma cells.

Our results showed that Hey1 is upregulated in melanoma tissues. Hey1 interference inhibited the migration and invasion of melanoma cells, while Hey1 overexpression exerted the opposite effects on melanoma cell behaviour and signaling transduction. We found that GRB2 and Hey1 have a similar expression trends. GRB2 is an adaptor protein and an important link in the receptor tyrosine kinase signaling cascade [21], providing a key association between cell-surface growth factor receptors and PI3K/AKT signal transduction [22]. Prior studies have shown that an imbalance in the GRB2 level in tumors can influence the invasion and metastasis of tumor cells in malignancies, such as in liver cancer and lung cancer $[23,24]$. Relatively, few studies have investigated the role of GRB2 in melanoma. One study showed that the cationic KT2 peptide downregulates GRB2 expression to inhibit the migration and invasion of human melanoma cells [25]. Another study indicated that phycocyanin hinders the proliferation of melanoma cells by downregulating GRB2/ERK signaling transduction [26]. However, we propose that Hey1 may augment the expression of GRB2 to promote the invasion and metastasis of melanoma cells. Notably, differential gene screening of human melanocytes of skins showed that GRB2 expression was downregulated during the differentiation and proliferation of human melanocytes [27], indicating that GRB2 is also linked to the malignant transformation of melanocytes.

The PI3K/AKT pathway is typically activated in melanoma [28]. These activation signals include receptor tyrosine kinases, and GRB2 provides a key link between PTKs and PI3K/AKT. When PI3K binds to GRB2 and is recruited to the cell membrane for activation by phosphorylation, AKT is then also recruited to the cell membrane and phosphorylated. Phosphorylated AKT acts on its target genes to regulate a variety of cellular processes [29]. P21 is a target gene of PI3K/AKT signaling pathway, to regulate various cellular processes including DNA repair, apoptosis, differentiation, cytoskeleton dynamics, cell migration, gene transcription, and reprogramming induced pluripotent stem cells. Interestingly, P21 not only plays an anti-cancer role but also generates a carcinogenic effect. This dual behaviour depends on its subcellular localisation, interaction partner, and phosphorylation state [30, 31]. We found that decreased phosphorylation of PI3K and AKT inhibited phosphorylation of P21, implying that Hey1 indirectly regulates the PI3K/AKT/P21 signaling network via GRB2.

Furthermore, previous studies revealed signaling crosstalks between the Notch and ERBB signaling pathways, which are activated simultaneously in melanoma to exert synergistic effects [32]. A review mentioned that Notch and ERBB signaling components (i.e., Notch1, Hey1, and ERBB3) are re-expressed in melanoma in a correlative manner, and proposed that the tumorigenesis and development of melanoma require two cascade functions [33]. Our study confirmed part of the hypothesis that Hey1, as the main component of Notch signaling, participates in the ERBB signaling pathway by activating the GRB2/PI3K/AKT signaling cascade.

In summary, we showed that Hey1 regulates the PI3K/AKT signaling pathway via GRB2 mediation. Hey1 promotes the EMT process, tumor matrix metalloproteinases expression, as well as the migration and invasion of melanoma cells. Nevertheless, whether Hey1 binds on the GRB2 promoter to regulate its expression remains further investigation. In conclusion, our study provides compelling evidence that Hey1 promotes melanoma cell migration and invasion through GRB2/PI3K/ AKT signaling cascade.

\section{Acknowledgements}

We thank the School of Laboratory Medicine, Chongqing Medical University for providing experimental platform.

\section{Ethics statement}

This study was approved by the Ethics Committee for Human Research, the the First Affiliated Hospital of Chongqing Medical University, and was conducted according to the approved guidelines. The patients whose tissues were used had provided written informed consent, in accordance with the Declaration of Helsinki.

\section{Funding}

This work was supported by the National Natural Science Foundation of China (81773307, 82073462 \& 82003384), Chongqing 271 Science and Technology Commission (cstc2018jcyjAX0195), Fundamental Research Funds for the Central 
Universities (2020CDJYGSG003), Chongqing Talents Program (CQYC2020058022), and Scientific Research Foundation from Chongqing University (02210011044110), China.

\section{Data Availability Statement}

The data used to support the findings of this study are included within the article, which are available from the corresponding author upon request.

\section{Competing Interests}

The authors have declared that no competing interest exists.

\section{References}

1. Longvert C, Saiag P. Melanoma update. La Revue de medecine interne. 2019; 40: 178-183.

2. Paluncic J, Kovacevic Z, Jansson PJ, Kalinowski D, Merlot AM, Huang ML, et al. Roads to melanoma: Key pathways and emerging players in melanoma progression and oncogenic signaling. Biochim Biophys Acta. 2016; 1863: 770-84.

3. Meierjohann S. Crosstalk signaling in targeted melanoma therapy. Cancer Metastasis Rev. 2017; 36: 23-33.

4. Weber D, Wiese C, Gessler M. Hey bHLH transcription factors. Curr Top Dev Biol. 2014; 110: 285-315.

5. Heisig J, Weber D, Englberger E, Winkler A, Kneitz S, Sung WK, et al. Target gene analysis by microarrays and chromatin immunoprecipitation identifies HEY proteins as highly redundant bHLH repressors. PLoS Genet. 2012; 8: e1002728.

6. Tsuru A, Setoguchi T, Matsunoshita $\mathrm{Y}$, Nagao-Kitamoto H, Nagano S, Yokouchi M, et al. Hairy/enhancer-of-split related with YRPW motif protein 1 promotes osteosarcoma metastasis via matrix metallopeptidase 9 expression. Br J Cancer. 2015; 112: 1232-1240.

7. Candy PA, Phillips MR, Redfern AD, Colley SM, Davidson JA, Stuart LM, et al. Notch-induced transcription factors are predictive of survival and 5-fluorouracil response in colorectal cancer patients. Br J Cancer. 2013; 109: 1023-1030.

8. Park SB, Hwang KT, Chung CK, Roy D, Yoo C. Causal Bayesian gene networks associated with bone, brain and lung metastasis of breast cancer. Clin Exp Metastasis. 2020; 37: 657-674

9. Yang YF, Wang YY, Hsiao M, Lo S, Chang YC, Jan YH, et al. IMPAD1 functions as mitochondrial electron transport inhibitor that prevents ROS production and promotes lung cancer metastasis through the AMPK-Notch1-HEY1 pathway. Cancer Lett. 2020; 485: 27-37.

10. Zhou Z, Cui D, Sun MH, Huang JL, Deng Z, Han BM, et al. CAFs-derived MFAP5 promotes bladder cancer malignant behavior through NOTCH2/HEY1 signaling. FASEB J. 2020; 34: 7970-7988.

11. Chen Y, Zou D, Wang N, Tan T, Liu Y, Zhao Q, et al. SFRP5 inhibits the migration and invasion of melanoma cells through Wnt signaling pathway. Onco Targets Ther. 2018; 11: 8761-8772.

12. Hodorogea A, Calinescu A, Antohe M, Balaban M, Nedelcu RI, Turcu G, et al. Epithelial-Mesenchymal Transition in Skin Cancers: A Review. Analytical cellular pathology. 2019; 2019:3851576.

13. Kessenbrock K, Plaks V, Werb Z. Matrix Metalloproteinases: Regulators of the tumor Microenvironment. Cell. 2010; 141: 52-67.

14. Xie J, Lin LS, Huang XY, Gan RH, Ding LC, Su BH, et al. The NOTCH1-HEY1 pathway regulates self-renewal and epithelial-mesenchymal transition of salivary adenoid cystic carcinoma cells. Int J Biol Sci. 2020; 16: 598-610.

15. Fukusumi T, Guo TW, Ren S, Haft S, Liu C, Sakai A, et al. Reciprocal activation of HEY1 and NOTCH4 under SOX2 control promotes EMT in head and neck squamous cell carcinoma. Int J Oncol. 2021; 58: 226-237.

16. Fukusumi T, Guo TW, Sakai A, Ando M, Ren S, Haft S, et al. The NOTCH4-HEY1 Pathway Induces Epithelial-Mesenchymal Transition in Head and Neck Squamous Cell Carcinoma. Clin Cancer Res. 2018; 24: 619-633.

17. Loureiro JB, Abrantes M, Oliveira PA, Saraiva L. P53 in skin cancer: From a master player to a privileged target for prevention and therapy. Biochim Biophys Acta Rev Cancer. 2020; 1874: 188438.

18. Tsitsipatis D, Klotz LO, Steinbrenner H. Multifaceted functions of the forkhead box transcription factors FoxO1 and FoxO3 in skin. Biochim Biophys Acta Gen Subj. 2017; 1861: 1057-1064.

19. Ding C, Tang W, Wu H, Fan X, Luo J, Feng J, et al. The PEAK1-PPP1R12B axis inhibits tumor growth and metastasis by regulating GRB2/PI3K/AKT signalling in colorectal cancer. Cancer Lett. 2019; 442: 383-395.
20. Leonardi GC, Falzone L, Salemi R, Zanghì A, Spandidos DA, Mccubrey JA, et al. Cutaneous melanoma: From pathogenesis to therapy (Review). Int J Oncol. 2018; 52: 1071-1080.

21. Tari AM, Lopez-Berestein G. GRB2: a pivotal protein in signal transduction. Semin Oncol. 2001; 28: 142-7.

22. Liao T, Jang H, Nussinov R, Fushman D. High-Affinity Interactions of the $\mathrm{nSH} 3$ / $\mathrm{cSH} 3$ Domains of GRB2 with the C-Terminal Proline-Rich Domain of SOS1. J Am Chem Soc. 2020; 142: 3401-3411.

23. Lv J, Zhang S, Wu H, Lu J, Lu Y, Wang F, et al. Deubiquitinase PSMD14 enhances hepatocellular carcinoma growth and metastasis by stabilizing GRB2. Cancer Letters. 2020; 469: 22-34.

24. Wang A, Wang J. E2F1-Induced Overexpression of Long Noncoding RNA SBF2-AS1 Promotes Non-Small-Cell Lung Cancer Metastasis Through Regulating miR-362-3p/GRB2 Axis. DNA and Cell Biology. 2020; 39: 1290-1298.

25. Maraming P, Klaynongsruang S, Boonsiri P, Peng SF, Daduang S, Rungsa P, et al. Anti-metastatic Effects of Cationic KT2 Peptide (a Lysine/Tryptophan-rich Peptide) on Human Melanoma A375.S2 Cells. In vivo. 2021; 35: 215-227.

26. Hao S, Li S, Wang J, Zhao L, Zhang C, Huang W, et al. Phycocyanin Reduces Proliferation of Melanoma Cells through Downregulating GRB2/ERK Signaling. J Agric Food Chem. 2018; 66: 10921-10929.

27. Mei X, Wu Z, Huang J, Sun Y, Shi W. Screening and analysis of differentially expressed genes of human melanocytes in skin cells mixed culture. American journal of translational research. 2019; 11: 2657-2667.

28. Lugović-Mihić L, Ćesić D, Vuković P, Novak Bilić G, Šitum M, Špoljar S. Melanoma Development: Current Knowledge on Melanoma Pathogenesis. Acta dermatovenerologica Croatica : ADC. 2019; 27: 163-168.

29. Michael AD.The role of the PI3K-AKT pathway in melanoma.Cancer J. 2012; 18: $142-7$.

30. Kreis NN, Friemel A, Ritter A, Roth S, Rolle U, Louwen F, et al. Function of p21 (Cip1/Waf1/CDKN1A) in Migration and Invasion of Cancer and Trophoblastic Cells. Cancers (Basel). 2019; 11: 989.

31. Xiao BD, Zhao YJ, Jia XY, Wu J, Wang YG, Huang F. Multifaceted p21 in carcinogenesis, stemness of tumor and tumor therapy. World J Stem Cells. 2020; 12: 481-487.

32. Zhang K, Wong P, Salvaggio C, Salhi A, Osman I, Bedogni B. Synchronized Targeting of Notch and ERBB Signaling Suppresses Melanoma tumor Growth through Inhibition of Notch1 and ERBB3. J Invest Dermatol. 2016; 136: 464-472.

33. Bedogni B. Notch signaling in melanoma: interacting pathways and stromal influences that enhance Notch targeting. Pigment Cell Melanoma Res. 2014; 27: $162-168$ 